INPLASY

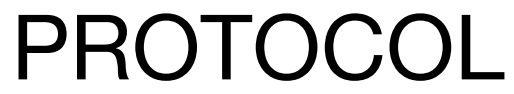

To cite: Zhao et al. Efficacy and safety of Shenmai injection in patients with chronic heart failure: a systematic review and metaanalysis. Inplasy protocol 2021120099. doi: 10.37766/inplasy2021.12.0099

Received: 22 December 2021

Published: 22 December 2021

Corresponding author:

Bo Li

dr.libo@vip.163.com

Author Affiliation:

Beijing Institute of Traditional Chinese Medicine/Beijing Hospital of Traditional Chinese Medicine.

Support: None.

Review Stage at time of this submission: Data analysis.

Conflicts of interest:

None declared.

\section{Efficacy and safety of Shenmai injection in patients with chronic heart failure: a systematic review and meta-analysis}

Zhao, GZ1; Liu, CY2; Wang, YB3; Li, B4.

Review question / Objective: To perform a systematic review and meta-analysis to assess the efficacy and safety of Shenmai injection as adjuvant treatment for chronic heart failure.

Condition being studied: Chronic heart failure.

Information sources: PubMed, Embase, the Cochrane Library, China National Knowledge Infrastructure (CNKI), Wanfang Database, and SinoMed.

INPLASY registration number: This protocol was registered with the International Platform of Registered Systematic Review and Meta-Analysis Protocols (INPLASY) on 22 December 2021 and was last updated on 22 December 2021 (registration number INPLASY2021120099).

\section{INTRODUCTION}

Review question / Objective: To perform a systematic review and meta-analysis to assess the efficacy and safety of Shenmai injection as adjuvant treatment for chronic heart failure.
Condition being studied: Chronic heart failure.

\section{METHODS}

Participant or population: patients diagnosed as CHF with NYHA classification from I to IV. 
Intervention: SMI as a complement to the standard treatments for CHF.

Comparator: Standard treatments alone.

Study designs to be included: Published randomized controlled trials (RCTs).

Eligibility criteria: The detailed inclusion criteria were summarized using PICOS approach (patients, intervention, comparisons, outcome, and study design type).

Information sources: PubMed, Embase, the Cochrane Library, China National Knowledge Infrastructure (CNKI), Wanfang Database, and SinoMed.

Main outcome(s): The primary outcomes included the clinical effective rate and LVEF level. The clinical effective rate was calculated by the following formula: (number of remarkable recovery participants + number of basic recovery participants)/total number of participants $x$ $100 \%$.

Additional outcome(s): The secondary outcomes included BNP level and the potency of lengthening the distance of 6min walk test (6MWT), and adverse events.

Quality assessment / Risk of bias analysis: Cochrane risk of bias tool.

Strategy of data synthesis: The statistical analysis was performed by Stata 12 (Stata Corp, College Station, TX). For dichotomous values, the risk ratios (RRs) and its $95 \%$ confidence intervals (Cls) were calculated. For continuous variables, weighted mean differences (WMDs) and its 95\% Cls were calculated.

Subgroup analysis: None.

Sensitivity analysis: None.

Country(ies) involved: China.

Keywords: chronic heart failure; Shenmai injection; Systematic review; Metaanalysis.
Contributions of each author:

Author 1 - Guozhen Zhao.

Author 2 - Chunyu Liu.

Author 3 - Yanbo Wang.

Author 4 - Bo Li. 